\title{
The bright future of x-ray sources: Linac-based free electron lasers
}

\author{
Ilka Flegel and Joerg Rossbach /DESY
}

$O$ February 22, 2000, an international team working at the TESLA Test Facility (TTF) at DESY, Hamburg, set a new record for the shortest wavelength of radiation ever achieved with a Free Electron Laser (FEL) [1]. The collaboration, involving around 140 scientists from 38 institutes in 9 countries, succeeded in generating ultraviolet radiation with a wavelength of $109 \mathrm{~nm}$. The previous best using this type of SASE (Self-Amplified Spontaneous Emission) free electron laser was $530 \mathrm{~nm}$ obtained by a group at Argonne [2]. Within a few weeks, the TTF team pushed the wavelength down to $80 \mathrm{~nm}$ and tuned the FEL to various wavelengths up to $180 \mathrm{~nm}$, demonstrating for the first time free wavelength tunability of SASE FELs over a large range.

To accomplish this decisive step towards shorter laser wavelengths, the TTF team made use of the electron beam from the superconducting linac of the TTF, set up for development and testing of new superconducting niobium cavities for DESY's planned 33-kilometre TESLA electron-positron linear collider. [3]

Having demonstrated SASE at wavelengths around $100 \mathrm{~nm}$, the collaboration will soon extend the TTF into a 300-metre device to operate in the soft X-ray range around $6 \mathrm{~nm}$ [4]. This facility should become available for user experiments by the end of 2003 .

The ultimate goal is to produce "hard" $\mathrm{X}$-rays with a wavelength of $0.1 \mathrm{~nm}$. Since this requires an electron beam of much higher energy, it is planned to integrate such a hard X-ray laser facility into the proposed TESLA linear collider.

\section{Synchrotron radiation}

Since its discovery in the mid-1940s, syn- chrotron radiation has evolved into an invaluable research tool for applications in many different fields of science - from surface physics, materials sciences and chemistry, to geophysics, molecular biology and medicine. Nowadays, third-generation synchrotron radiation sources produce high brightness photon beams covering the complete range from infrared to hard X-rays. However, the electrons producing these photons do not radiate in phase, and a true high-power X-ray laser has so far remained a dream.

How to reach this "ultimate" X-ray source has been the subject of many efforts, both theoretically and experimentally, over the last twenty years. During the past six to eight years, a consensus has developed that fourth-generation sources, implying a higher degree of coherence, higher power, brilliance, and ultrashort pulses, possibly at very short wavelengths in the hard X-ray region, would probably involve a linear accelerator driving a free electron laser.

This shift from storage rings to linear accelerators is necessary, because the quality of the electron beam - short bunch lengths and small beam emittance - is limited in storage rings, but crucially important for the FEL process. For the SASE

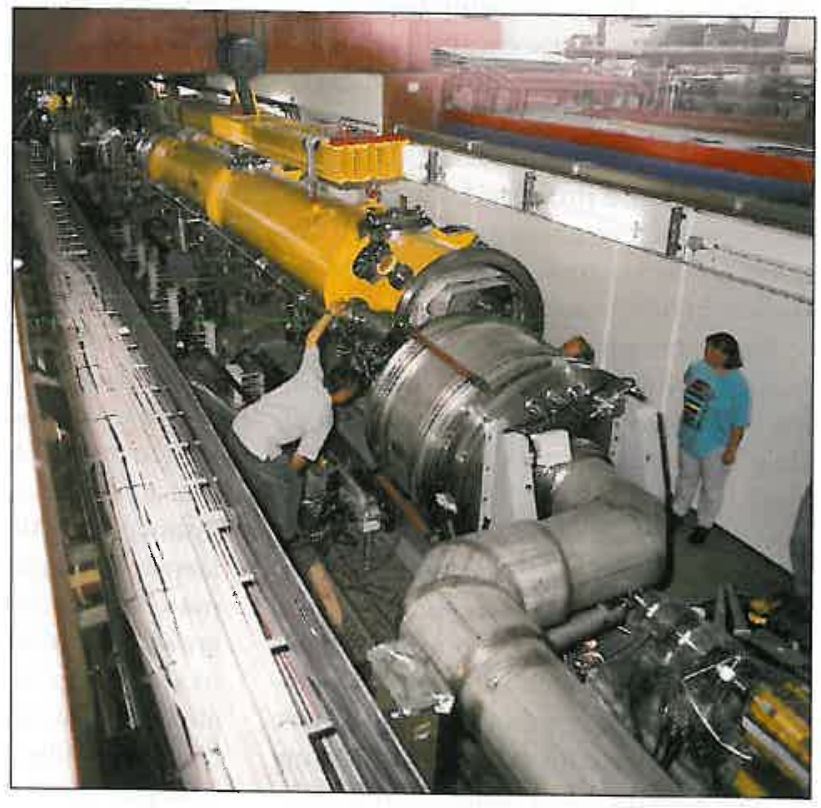

Fig 1: An international team using the superconducting linac at the TESLA Test Facility (TTF) at DESY, Hamburg, has set a new record for the shortest wavelength of radiation ever achieved with a Free Electron Laser (FEL) - Photo DESY. concept at short X-ray wavelengths the limitations of storage rings become particularly noticeable.

\section{Free electron lasers}

The main ingredients of a free electron laser are a high-energy electron beam with very high brightness and a periodic transverse magnetic field, such as that produced by an undulator structure. As the electron bunches wiggle through the magnetic field, they emit synchrotron radiation around their direction of motion. For small undulations, the radiation is quasimonochromatic. For every undulator period, the radiation phase moves ahead of 
the electrons by a distance equal to this specific resonance wavelength - keeping each electron in phase with the radiation field.

Depending on the relative phase between radiation and electron oscillation, electrons experience either a retardation or acceleration with respect to the mean electron velocity. To obtain SASE, the electron beam has to be of sufficient quality and the undulator long enough. In this case, the electron bunch starts to develop a longitudinal density modulation with a period length equal to the resonance wavelength of the undulator. This electron density modulation or "microbunching" reduces phase cancellation in the emission process, increasing the intensity of the emitted radiation. This radiation interacts further with the electron beam and enhances the bunch density modulation, thereby further increasing the intensity. The net result is an exponential increase of radiated power to a saturation value that is approximately six orders of magnitude higher than the power of conventional undulator radiation.

\section{No mirrors}

Like conventional lasers, most present free electron lasers use an optical cavity formed by mirrors to store the light from many successive electron bunches. Many of these FELs work in the infrared range, and some even reach ultraviolet wavelengths. However, extending them into the $\mathrm{X}$-ray regime is difficult due to the lack of well reflecting surfaces at wavelengths below $150 \mathrm{~nm}$ and the increasing risk of radiation damage.

An alternative path to shorter wavelengths was found with the development of SASE free electron lasers. They start from noise and reach saturation within a single pass of a high-brightness electron bunch through a very long undulator, without any mirrors.

The concept of SASE free electron lasers was introduced in the early 1980s [5], and further explored in 1984 [6], soon leading to first experimental tests [7]. In 1997-98, a Los Alamos/UCLA experiment at Los Alamos [8] produced a gain of $3 \times 10^{5}$ for the first time, and established the proofof-principle of SASE theory at a wavelength of 12 micrometres. Recently, SASE at $530 \mathrm{~nm}$ was demonstrated at Argonne [2] and saturation power was reached a few months later [9].

\section{Testbed}

The TESLA Test Facility (TTF) was set up at DESY in 1993 to provide a testbed for

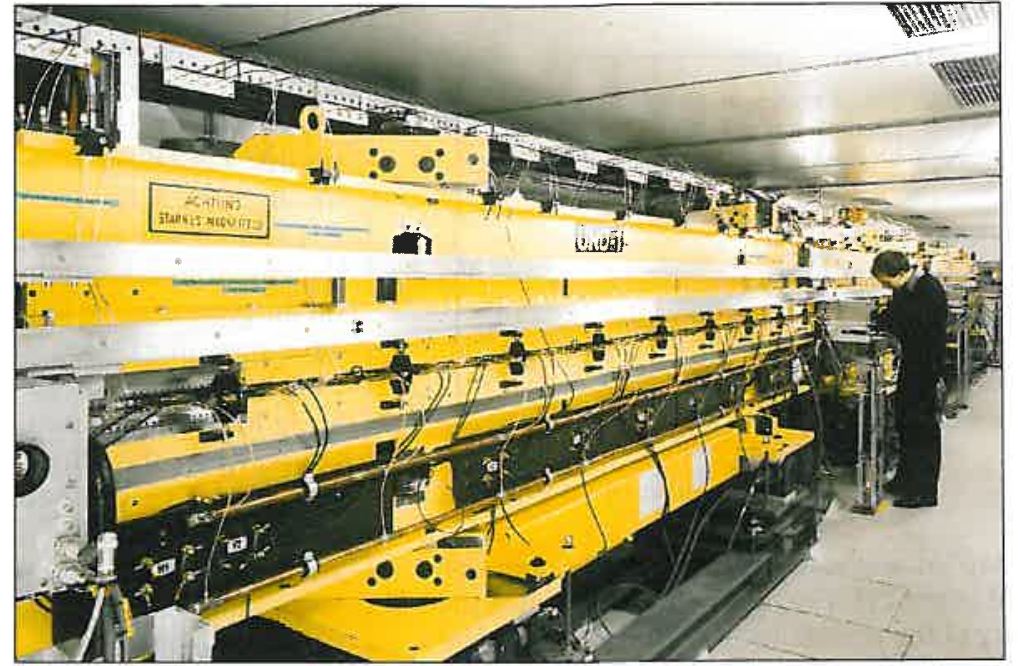

Fig 2: The undulator used in DESY's TTF free electron laser is a 15-metre magnetic structure comprising three 4.5-metre modules, each made up of 652 permanent magnets. To ensure that the electron beam does not deviate from a straight line by more than $\mathbf{5 0}$ micrometres, the magnets have to be fitted together to an accuracy of just a few microns. In addition, the undulator is set up in a special air-conditioned hutch where the temperature is held constant with a precision of 0.1 degrees in order to avoid expansion of undulator components.

the TESLA linear collider project, especially the superconducting niobium cavities for particle acceleration. In 1994, work began on the test accelerator which will finally be extended into a 300-metre soft $\mathrm{X}$-ray FEL comprising all the basic elements that will be subsequently employed in the hard X-ray lasers integrated into TESLA.

In the first phase, operating around 100 $\mathrm{nm}$ wavelength, the TTF is equipped with a $15 \mathrm{~m}$ long undulator, a bunch compres- sor reducing the bunch length thus increasing the peak current, and a radiofrequency (RF) photocathode electron gun. There are essentially two technical challenges to be met for an X-ray FEL. First, it is crucial to generate and accelerate a low emittance and high peak current electron beam. This can be achieved using a highbrightness radiofrequency photocathode gun as an electron source. The electron gun currently used at the TTF-FEL is a joint contribution of Fermilab, INFN/Mi-

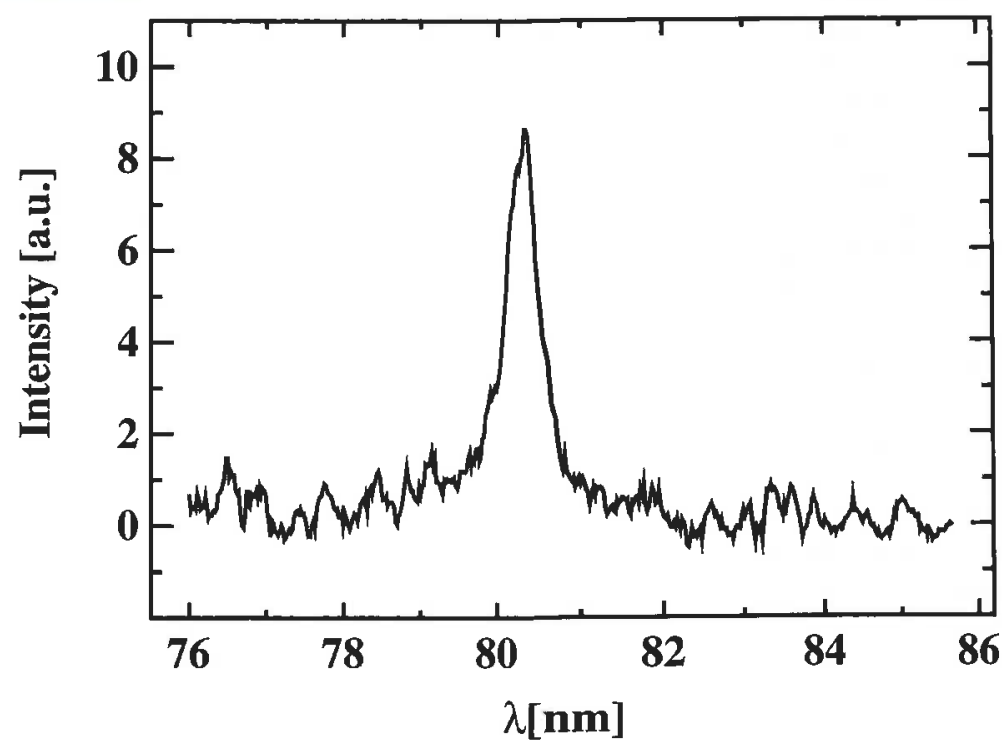

Fig 3: Spectrum of FEL radiation at a wavelength of 80 nanometres. 


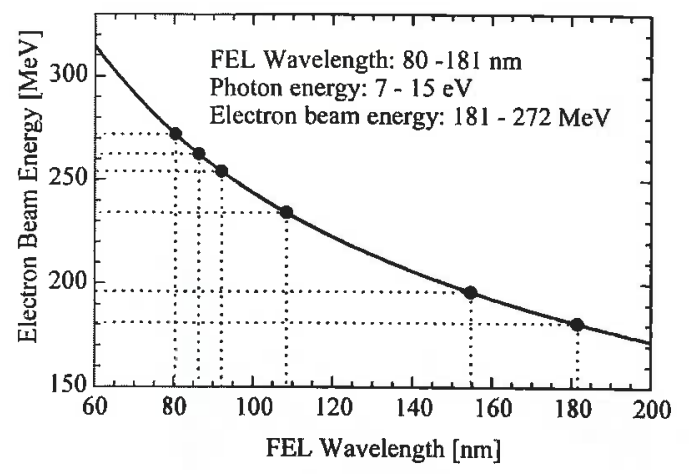

Fig 4: "Map" of wavelengths of SASE FEL radiation obtained at the TTF. The picture demonstrates the tunability of the wavelength which is achieved by a variation of the electron energy in the accelerator. high-energy physics facility with operation of a radiation source continues a long and fruitful tradition at DESY. Technically, both X-ray SASE FELs and linear colliders depend fundamentally on the generation of lowemittance, short electron bunches and on accelerating long bunch trains conserving the beam properties. This is best achieved with a superconducting linac, combining high accelerating gradients and low wakefield effects with long bunch trains at high duty

lan, Rochester, the Max Born Institute in Berlin, and DESY.

It has meanwhile been demonstrated that such a particle source can drive a facility 24 hours a day for weeks and even months. Since the RF gun performance is so critical for the further development, DESY is building up a stand-alone gun test facility at its institute in Zeuthen near Berlin.

The peak current of the electron bunches produced by the low-emittance gun is still not high enough to reach laser saturation within an undulator of reasonable length. The solution is to compress the bunches longitudinally to increase the peak current. This can be achieved by using a "bunch compressor chicane" - a sequence of deflecting magnets.

The principle is not new, but it is a challenge to reach a few kilo-amperes of peak current since this requires bunch lengths below $0.1 \mathrm{~mm}$. Accelerating the beam off the crest of the RF wave in the linac creates an energy-phase correlation that can be used to shorten the bunch. When passing the chicane, electrons with different momenta travel different path lengths. The TTF-FEL currently uses a bunch compressor at $140 \mathrm{MeV}$ which compresses the bunch length below $0.5 \mathrm{~mm}$

rms.

The second important technical challenge is to keep the electron beam (focused to a transverse beam size of about $0.1 \mathrm{~mm}$ ) in essentially complete overlap with the photon beam as it passes through the undulator. This sets new standards for undulator alignment procedures and beam orbit control.

\section{Interleaved operation}

Combining the machine expertise at a cycle, due to low power losses.

For power cost reasons, a superconducting linear collider has an RF-on-time fraction of only $1 \%$. Consequently, there is room for further RF pulses to accelerate an interleaved electron beam for FEL operation. In this way, the most expensive component of an X-ray laser, the linac, is shared with the high-energy physics community.

All TTF findings are consistent with existing models for SASE FELs. So far, a laser gain of a few thousand has been observed, while laser saturation is expected well beyond $10^{6}$. Thus, the next steps will be focused on achieving higher laser gain by improving orbit control and electron beam quality. Operation with long trains of several thousand electron bunches will also be tested. The TESLA collaboration will then upgrade the superconducting linac to $1 \mathrm{GeV}$, bringing the FEL wavelength down to $6 \mathrm{~nm}[4]$. The new user facility should be ready for experiments by the end of 2003. As for the TESLA Linear Collider with Integrated X-ray Lasers, a conceptual design was published in 1997 [3], and a Technical Design Report, including schedule and costs, will be presented in 2001 for evaluation by the German Science Council (Wissenschaftsrat), the German Federal Government's scientific advisory board. As a first step towards formal planning permission, an agreement between the relevant German federal states was signed in 1998.

The above article is an updated version of an article that appeared in CERN Courier, July/August 2000, pp. 26-28.

\section{References:}

[1] J.Andruszkow et al., "First Observation of Self-Amplified Spontaneous Emission in a Free-Electron Laser at $109 \mathrm{~nm}$ Wavelength", accepted for publication in Phys. Rev. Lett., scheduled for Vol. 85 (17), Oct. 23,2000

[2] S.V. Milton et al., "Observation of SelfAmplified Spontaneous Emission and Exponential Growth at $530 \mathrm{~nm}$ ", Phys. Rev. Lett. 85, 988-991 (2000)

[3] R. Brinkmann, G. Materlik, J. Rossbach and A. Wagner (eds.), "Conceptual Design of a $500 \mathrm{GeV} \mathrm{e}^{+} \mathrm{e}^{-}$Linear Collider with Integrated X-ray Laser Facility", DESY 1997 048 and ECFA 1997-182 (1997)

[4] T. Åberg et al., "A VUV FEL at the TESLA Test Facility at DESY", Conceptual Design Report, DESY Print TESLA-FEL 9503 (1995)

[5] A.M. Kondratenko and E.L. Saldin, "Generation of Coherent Radiation by a Relativistic Electron Beam in an Ondulator", Part. Accelerators 10, 207-216 (1980)

[6] R. Bonifacio, C. Pellegrini, and L. Naducci, "Collective Instabilities and High Gain Regime in a Free Electron Laser", Opt. Commun., 50, 373-378 (1984)

[7] E.T. Scharlemann, W. M. Fawley, B.R. Anderson, and T.J. Orzechowski, "Comparison of the Livermore Microwave FEL Results at ELF with 2D Numerical Simulations", in Proceedings of the Seventh FEL Conference (FEL86), Nucl. Instrum. and Meth. A 250, 150-158 (1986)

[8] M.J. Hogan, C. Pellegrini, J. Rosenzweig, S. Anderson, P. Frigola, A. Tremaine, C. Fortgang, D.C. Nguyen, R.L. Sheffield, J. Kinross-Wright, A. Varfolomeev, A.A. Varfolomeev, S. Tolmachev and R. Carr, "Measurements of Gain Larger then $10^{5}$ at $12 \mathrm{~mm}$ in a Self-Amplified SpontaneousEmission Free-Electron Laser", Phys. Rev. Lett. 81, 4867-4870 (1998)

\section{[9] see: http://www.aps.anl.gov/aod/} mcrops/leutl/Saturation000923.html 